\title{
Critical Care of the Liver Transplant Recipient
}

\author{
Thomas M. A. Fernandez ${ }^{1} \cdot$ Paul J. Gardiner ${ }^{2}$
}

Published online: 30 September 2015

(c) Springer Science + Business Media New York 2015

\begin{abstract}
Patient survival following orthotopic liver transplantation has greatly increased following improvements in surgical technique, anesthetic care, and immunosuppression. The critical care of the liver transplant recipient has paralleled these improvements, largely thanks to input from multidisciplinary teams and institutionspecific protocols guiding management and care. This article provides an overview of the approach to critical care of the postoperative adult liver transplant recipient outlining common issues faced by the intensivist. Approaches to extubation and hemodynamic assessment are described. The provision of appropriate immunosuppression, infection prophylaxis, and nutrition is addressed. To aid prompt diagnosis and treatment, intensivists must be aware of postoperative complications of bleeding, primary nonfunction, delayed graft function, vascular thromboses, biliary complications, rejection, and organ dysfunction.
\end{abstract}

Keywords Orthotopic liver transplantation - Critical care . Intensive care unit · Hemodynamic monitoring ·

Immunosuppression · Primary nonfunction - Delayed graft function $\cdot$ Hepatic artery thrombosis

This article is part of the Topical Collection on Critical Care Anesthesia.

Thomas M. A. Fernandez

tomf@adhb.govt.nz

1 Department of Anesthesia and Perioperative Care, Auckland City Hospital, 2 Park Road, Grafton, Auckland 1023, New Zealand

2 Department of Critical Care Medicine, Auckland City Hospital, Auckland, New Zealand

\section{Introduction}

Orthotopic liver transplantation (OLT) remains the only curative modality for patients with end-stage liver disease. Since the first liver transplant in 1963, significant advances have occurred in the intensive care unit (ICU) management of patients, largely due to innovations in surgical technique, advances in anesthesia and perioperative care, and improvements in immunosuppression. Patients now experience shorter ICU stays, fewer readmissions, and improved overall survival.

Indications for liver transplant and patient presentations vary, each influencing the immediate postoperative course. Transplant surgery occurs in specialized centers following rigorous work-up by multidisciplinary teams who go on to play a role in the patient's ongoing care. The initial ICU postoperative period following transplantation is influenced by a number of factors: the patient's underlying pathology, disease severity, comorbidities, quality of donor graft, and the intraoperative course [1]. All must be taken into account by the intensivist.

Following a thorough handover and stabilization, attention is given to assessment of adequate graft function, provision of immune and infection prophylaxis, and adequate nutrition. Intensivists must be aware of complications that may initially arise in the ICU requiring early detection and management. Attention to detail with adherence to institution-specific evidence-based protocols is helpful in reducing ICU length of stay, readmission, and overall morbidity and mortality.

\section{Handover}

Of great importance when admitting a liver transplant recipient is the information received at handover. The quality of the donor liver and the intraoperative course have great implication on immediate postoperative care. 


\section{Donor Organ Quality}

While the optimal deceased donor organ is that of a sizematched previously healthy donor following fatal brain injury [2], organ supply shortages and growing transplant waiting lists have led to the increased use of marginal donors. The donor risk index [3] identified donor characteristics predictive of increased risk of graft failure thereby allowing better determination of donor-recipient pairing suitability. In addition, a prolonged warm ischemia time, donation after circulatory death, macrosteatosis $>30 \%$, advanced donor age, and donor hypernatremia have been described as increasing the risk of impaired allograft function, rejection, and primary nonfunction [1]. Ultimately the intensivist's knowledge of donor organ quality proves valuable in anticipating potential pitfalls and predicting poor function.

\section{Intraoperative Course}

The intraoperative course is a useful predictor of initial intensive care course. Surgeons are questioned on graft quality, their assessment of function, operative anatomy, surgical approach, and anticipated concerns. The anesthesia team conveys features pertinent to the overall care of the patient, including blood loss, inotropic support, product replacement, coagulation status, and complications or instability. Intraoperatively, acid clearance, hemodynamic stability, glucose and bile production, and improvement in coagulation are all initial signs of stable and successful graft function. With this information in hand, attention may be turned to assessment and care of the patient.

\section{Patient Assessment and Care}

As with any admission to the ICU, an overall assessment of airway, breathing, and circulation must be performed. A systematic approach is particularly important for liver transplant recipients who have potential for instability within any of these domains.

\section{Postoperative Ventilation/Extubation}

In assessment of "airway and breathing," and in conjunction with the operative team, the intensivist determines the need for ventilation and timing of extubation. The practice of immediate extubation in the operating room versus later in the ICU, varies among transplant centers. Once considered unconventional, a number of centers now adopt a "Fast Track" approach to initial postoperative care with immediate or early extubation for patients meeting defined criteria [4-7]. Others advocate a period of postoperative ventilation until assurance of hemodynamic stability, confirmation of graft function, and exclusion of need for return to the operating room.

Proponents of immediate/early extubation cite numerous advantages including reduced length of ICU and hospital stay, earlier return of graft function, and lower costs and resource consumption [8]. Disadvantages to postoperative ventilation include increased risk of ventilator-associated lung injury and pneumonia, worsening venous congestion of the graft, and reduction in splanchnic flow. Mandell et al. found no negative impact on long-term outcome from early extubation, with 1- and 3-year survival rates remaining above national average [5]. Furthermore, a lack of evidence exists showing that routine postoperative ventilation actually improves outcome [9].

Opponents of immediate extubation cite the practice as unsafe, adding unnecessary risk. Studies have yet to show overall patient or graft survival is improved by this practice [10]. Additionally, success is institution- and resourcespecific. An existing multi-center trial indeed showed wide variation in successful extubation rates (5-67\%) despite attempts at uniform criteria $[4,11]$.

Agreement exists on the benefit of maintaining a period of mechanical ventilation for patients with significant encephalopathy, hypoxemia, hemodynamic instability, unstable coagulopathy, large volume product administration, high-risk of graft dysfunction, respiratory complications, and increased risk for return to surgery.

Ultimately, each center must follow its own protocol regarding timing of extubation. A selective approach may be best, with emphasis on avoiding unnecessary delay in time to extubation. Patients should be extubated if stable, with adequate graft function ascertained and the need for emergent return to the operating room having been excluded [10].

\section{Hemodynamic Assessment and Optimization}

The assessment of "circulation" and hemodynamics in liver transplant recipients is complex, often influenced by bleeding, third spacing, intraoperative resuscitation, and an underlying high-output, vasodilated state. In assessing patients, the intravascular volume status, underlying cardiac function and systemic vascular resistance (SVR) must be considered [1]. Assessment should commence with thorough clinical examination taking into account hemodynamic parameters, blood loss, product/fluid replacement, and urine output. Patient comorbidities such as renal dysfunction, cardiac failure, pulmonary hypertension, and right ventricular failure add to the complexity. Assessment and cessation of any ongoing bleeding should be ensured at this point.

A variety of hemodynamic monitoring tools exist though use varies and is often influenced by those utilized 
intraoperatively. Arterial blood pressure monitoring and central venous line insertion are standard. Agreement exists, however, that central venous pressure is an unreliable indicator of intravascular and stroke volume [12, 13].

Transesophageal echocardiography provides real-time monitoring of preload status, cardiac contractility, and the differentiation of various cardiac pathologies. Its use in the ICU is limited by cost, availability, the need for expertise in interpretation, and the potential for esophageal or variceal injury/bleeding from probe insertion.

Pulmonary artery catheters (PAC) provide information on cardiac filling pressures. Once common place, their use is decreasing and controversial, as insertion is not without risk [14] and numerous studies have shown wedge pressure to be a poor predictor of fluid responsiveness [13, 15, 16•]. Mixed venous oxygen saturation allows an indirect measure of cardiac output, and some PAC devices utilize rapidresponse thermistor calculation of continuous cardiac output and right ventricular end-diastolic volume. A significant advantage of the PAC lies in its ability to directly measure pulmonary arterial pressures and thereby guide management of high-risk patients with portopulmonary hypertension.

Arterial pressure waveform analysis provides continuous measurement of stroke volume variation and pulse pressure variation to enable assessment of fluid responsiveness and cardiac output. Advantages include ease of interpretation, continuous beat-to-beat analysis, and minimal invasiveness. Limitations, however, include varied reliability during hemodynamically unstable conditions, particularly when vascular resistance changes to a large extent, as occurs with OLT recipients [17].

Hemodynamic optimization following OLT should ensure adequate cardiac filling, central blood volume, and end organ perfusion. Avoidance of excessive fluid is essential to prevent pulmonary congestion, subsequent graft edema, third spacing, and capillary leak [1]. While cirrhotic patients exhibit a hyperdynamic, high cardiac output state, some have poor cardiac contractility which may manifest following transplantation. Optimizing oxygen delivery through adequate cardiac output should be the principal focus with caution to avoid over resuscitating or under resuscitating the unstable patient [18].

Blood transfusion to maintain hemoglobin between 8 and $10 \mathrm{~g} / \mathrm{dL}$ and hematocrit $25-30 \%$ achieves adequate oxygen delivery to the graft [19] while reducing risk of vessel thrombosis from increased viscosity at higher hemoglobin levels. Vasopressor support maintains organ perfusion in patients with inadequate mean arterial pressure despite adequate volume replacement. Choice of agent varies and should be tailored to the patient. Norepinephrine is commonly utilized due to the low SVR state of end-stage liver disease patients.
An accurate fluid balance is necessary as abnormalities may occur from massive fluid shifts, prolonged surgery, and ongoing bleeding. In the hemodynamically stable recipient, an initial negative fluid balance may decrease early pulmonary complications and improve oxygen delivery to the graft [20]. In a retrospective review by Levy et al., cardiopulmonary dysfunction was the primary etiology of ICU readmission following OLT, with fluid overload as the main cause on subgroup analysis [21]. Recipients with significant renal dysfunction requiring renal replacement therapy (RRT) necessitate even stricter fluid balance for prevention of volume overload while maintaining perfusion.

\section{Graft Function}

Assessment of graft function is required early in the ICU admission in order to detect and correct any dysfunction. Assessment is based on clinical, laboratory, and radiological parameters.

Clinically, adequate graft function is determined by stabilization of hemodynamics, production of bile, an increasing urine output and improving mentation with reversal of encephalopathy. An unexplained failure to reduce vasoactive support is often indicative of poor graft function.

Lab assessment shows improving acid-base status, decreasing lactate, rising blood glucose (often requiring insulin), and improvement of coagulation with normalization of prothrombin time. Serial transaminase levels assess hepatocyte death and display a rise and peak (due to preservation injury) during the first $24-48 \mathrm{~h}$ before falling [1]. A persistent elevation or significant rise in AST and ALT suggests parenchymal injury warranting further investigation. Bilirubin, alkaline phosphatase (ALP), and gamma glutamyl transpeptidase (GGT) assess hepatic secretory function. They can remain normal up to day 5 before peaking day 7 to 14 due to reperfusion injury [22].

Radiological assessment of graft function involves routine postoperative doppler ultrasonography to determine graft inflow hemodynamics (patency of hepatic artery and portal vein) and outflow hemodynamics (hepatic vein). If vascular compromise is detected, surgical exploration or angiography may be warranted to correct underlying pathology.

\section{Immunosuppression}

Improvements in immunosuppression have made the greatest impact on survival rates following liver transplantation. Most centers commence calcineurin inhibitors (CNI) and corticosteroids with or without an anti-proliferative agent. Induction antibody therapy is increasingly 
utilized where a period of CNI-free treatment is desirable. In determining the appropriate regimen, intensivists are aided by hepatologists and institution-based protocols. Consensus among transplant centers has yet to be reached.

\section{Primary Immunosuppression}

Tacrolimus (a CNI that inhibits interleukin-2 (IL2) and interferon gamma) has become the mainstay immunosuppression agent. Superiority in preventing acute rejection, steroid-resistant rejection, graft loss and postoperative death $[23,24]$ makes this the first line agent, replacing cyclosporine. Side effects include diabetes, hypertension, nephrotoxity, hyperkalemia, hemolytic uremic syndrome, neurotoxicity and nausea/vomiting. The potential for drug interactions must be considered given it is cytochrome P-450 metabolism. Dosage should be guided by therapeutic drug monitoring with higher initial target levels for the first 6-8 weeks post transplant [25] and subsequent lower levels in the event of no rejection. Target ranges differ between transplant centers.

Corticosteroids remain a cornerstone of initial immunosuppression protocols with an additional role during acute rejection. Weaning patients where possible avoids side effects of diabetes, fluid retention, hypertension, delirium, osteoporosis, poor wound healing, and adrenal suppression. Steroids have also been implicated in hepatitis $\mathrm{C}$ recurrence [26]. Most centers utilize $500 \mathrm{mg}$ or $1 \mathrm{~g}$ methylprednisone at induction with tapered lower intravenous (IV) doses postoperatively until eating. A period of oral prednisone at slowly reducing doses is then given over 3-6 months. Steroid-free immunosuppression has been shown possible [27] and may benefit patients with hepatitis C, diabetes, and hypertension.

\section{Adjuvant Immunosuppression}

Mycophenolate and azathioprine are antimetabolites that selectively inhibit T-cell and B-cell proliferation. Their role lies in reduction or discontinuation of CNIs to treat side effects of renal and neurological toxicity. Azathioprine is preferentially used in pregnancy due to increased safety experience.

Sirolimus and everolimus are mammalian target of rapamycin (mTOR) inhibitors that prevent T-cell proliferation by inhibiting IL-2 transduction causing cell cycle arrest at the $\mathrm{G} 1$ to $\mathrm{S}$ phase. Initial concerns were raised regarding risk of hepatic arterial thrombosis resulting in an FDA black box warning [28]. Subsequent studies have disproven this, though avoidance of early and large loading doses is still advised [25]. Their utility lies in their safety in renal dysfunction. Other side effects include leukopenia, thrombocytopenia, hypercholesterolemia, anemia, and gastrointestinal dysfunction.

Antibody therapy plays a role in steroid-resistant rejection and as CNI-sparing agents for patients with pretransplant renal failure. Basiliximab is a chimeric mousehuman monoclonal antibody against the IL-2 receptor that prevents T-cell proliferation [29]. Polyclonal antibody therapies such as antithymocyte globulin are less commonly used following liver transplantation. They are usually only considered in the setting of recurrent steroidresistant rejection.

\section{Infection Prophylaxis}

Liver transplant recipients carry a high infection risk, contributed to by immunosuppression, poor nutritional status, prolonged hospitalization, and the presence of catheters, lines, and drains. Sepsis represents the leading cause of critical illness, morbidity, and 1-year mortality in liver transplantation $[22,30]$. The intensivist's role lies in ensuring attention to strict infection control and provision of appropriate prophylaxis and treatment where necessary.

Infections include surgical site infections, pneumonias, catheter-related bloodstream infections, urinary tract infections, abdominal collections, and cholangitis. Risk is increased by acute liver failure, reoperation, and complicated biliary anastomosis [30]. Treatment often involves cautious reduction of immunosuppression, identification of causative organisms, and anti-infectious therapy.

Prophylaxis covering bacterial, fungal, and viral pathogens varies among centers. Antibacterial prophylaxis utilizes pre-incision IV antibiotics for surgical site infection with broad spectrum gram-positive and gram-negative coverage $24 \mathrm{~h}$ postoperatively. Broader coverage (e.g., meropenem and vancomycin) may be initiated for higher risk patients. Trimethoprim-sulfamethoxazole is given as prophylaxis for Pneumocystis jiroveci pneumonia.

Candida is the predominant fungal infection following liver transplantation; however, Aspergillus infection and others may also occur. Most centers utilize oral fluconazole or liposomal amphotericin B for high-risk recipients (i.e., renal failure, acute liver failure, prolonged preoperative hospitalization, large transfusion requirements, reoperation, or retransplantation) [31].

Herpes simplex virus reactivation and cytomegalovirus (CMV) are early opportunistic viral infections following transplant [22, 30]. Prophylaxis has lead to reduction in incidence. Management approach varies including universal prophylaxis, prophylaxis for high-risk patients only (e.g., CMV positive donor organ), or preemptive therapy in established infection [22]. IV ganciclovir and oral valganciclovir are mainstays of antiviral therapy. 
The presence of infection following liver transplantation may lead to poor graft function, prolonged ICU stay, and increased risk of multi-organ failure [19]. Drug interactions between anti-infectious agents and immunosuppressants often increase complexity of management. Ultimately adherence to established protocols with appropriate input from transplant infectious disease specialists is key [32].

\section{Nutrition}

Attention to nutritional support following liver transplantation is essential in the ICU to aid postoperative recovery. Malnutrition contributes to infection, respiratory complications, prolonged ICU stay, and decreased survival [33]. Liver transplant patients have diverse abnormalities of carbohydrate, lipid, and protein metabolism [19], so specialist dietician input is fundamental.

Nutritional therapy should ensure adequate protein, calories, vitamins, and minerals without contributing to pre-existing complications of ascites, encephalopathy, or metabolic derangement [34]. The catabolic state of the post-liver transplant patient necessitates protein intake of $1.5-2.0 \mathrm{~g} / \mathrm{kg}$ dry weight $[19,34,35]$. The presence of encephalopathy requires enrichment of branched chain amino acids rather than reduction in protein content.

Some centers encourage immediate return to oral feeding if permitted by gastrointestinal (GI) function. Nasojejunal or nasogastric feeding is utilized in the early postoperative period when delays in return of GI function occur. Total parenteral nutrition is usually unnecessary and avoided.

While immediate postoperative nutrition is aimed at correcting deficiencies, long-term management should be tailored to prevent common posttransplant metabolic complications of diabetes, obesity, hypercholesterolemia, and osteoporosis. Continued input from dietitians on discharge from ICU is therefore necessary.

\section{Postoperative Complications}

The postoperative liver transplant recipient faces a number of potential early complications. Responsibility for initial detection, prevention, and management lies with the intensive care team. Common high-risk early complications include bleeding, graft nonfunction/dysfunction, vascular thrombosis, rejection, biliary complications, and respiratory, renal, or neurological dysfunction.

\section{Bleeding/Coagulopathy}

Most bleeding complications present early in the ICU admission with management being part of initial hemodynamic stabilization. End-stage liver disease alters primary hemostasis, coagulation, and fibrinolysis. Coagulopathy may be predicted from the patient's operative course and laboratory coagulation parameters. Hypothermia, acidosis, and hypocalcemia should be avoided. Blood products are continued where clinically indicated while awaiting return of graft function. In situations of treatmentresistant nonsurgical bleeding, the off-label use of factor VIIa (90 IU/kg) has been shown to be of potential benefit [36]. Etiological factors leading to bleeding include underlying poor graft function and coagulopathy, massive transfusion, poor hemostasis, inadequate product replacement, heparin release from the implanted graft, and postreperfusion syndrome [19]. In patients with portal hypertension, fibrinolysis and thrombocytopenia may also be contributing factors.

Management of coagulopathy and transfusion practice varies. The use of thromboelastography (TEG) or rotational thromboelastometry allows point-of-care assessment of coagulation and the ability to correct specific deficiencies [37•]. Studies have shown reduction in product transfusion through TEG with less allogenic blood product exposure $[38,39]$. Evidence also exists for TEG-guided detection of hypercoagulable states with reduction in thrombotic complications [40]. Despite this, consensus is lacking on how best to monitor and manage bleeding following liver transplant [37•] with $<30 \%$ of centers using viscoelastic tests routinely [41].

Overall, a delicate and complex balance between bleeding and thrombosis exists in the liver transplant recipient [42•]. The patient's underlying clinical condition must be taken into account. Ultimately, applying clinical judgment to each presentation and "treating the patient" is fundamental.

\section{Primary Graft Nonfunction}

Primary graft nonfunction (PNF) is a rare and catastrophic event associated with increased length of ICU stay, morbidity, and mortality. It is often immediately evident presenting with hemodynamic instability, acute renal failure, treatment-resistant coagulopathy, hypoglycemia, and lactic acidosis [43]. Terminology surrounding graft dysfunction varies. The United Network for Organ Sharing defines PNF as irreversible graft function requiring retransplantation within 7 days of implantation. Graft failure requiring retransplantation after 7 days can be termed delayed PNF. Petrowski and Busuttil categorize risk factors into donor, procurement, and recipient related [44]. Donor factors include advanced donor age, liver macrosteatosis, and warm ischemia after circulatory death. Procurement factors include poor organ flush or cooling after cross clamp, inappropriate preservation, and cold ischemia $>12 \mathrm{~h}$. 
Recipient factors are poor vascular inflow, prolonged warm ischemia, retransplantation or cardiac arrest after reperfusion [44-46]. It is important to differentiate PNF from delayed graft function which eventually improves.

\section{Delayed Graft Function}

Delayed graft function that progressively improves (also known as initial poor graft function) refers to dysfunction not resulting in retransplantation or death. While no universally accepted definition or criteria exits, all generally include an elevated aspartate aminotransferase (AST)/alanine aminotransferase (ALT) $>2000$ units/L within 7 days. Presentation is similar to PNF with central nervous system (CNS) changes, resistant coagulopathy, oliguria, hypoglycemia, and elevated transaminases. Treatment is supportive involving administration of FFP, dextrose to control hypoglycemia, hemodynamic support, and correction of metabolic disturbances. Novel supportive therapies have been described using prostaglandin E1, N-acetylcysteine, and liver assist devices [19, 44]. Although, in most cases, graft function returns to normal, close clinical observation is required to monitor for progression to PNF or delayed PNF requiring retransplantation [44].

\section{Vascular Thrombosis}

Hepatic artery thrombosis (HAT) is a complication with potential for graft loss, morbidity, and prolonged ICU stay. Early doppler ultrasonography is routinely used to evaluate artery patency. Contributing factors include hemoconcentration, hypercoagulability, poor arterial flow, increased sinusoidal resistance, and anastomotic stenosis [19]. Divided into early and late thrombosis, the former usually presents with problems related to graft function. Late thrombosis ( $>30$ days post transplant) [47•] often presents with biliary complications and a milder clinical course. HAT has been reported to occur in up to $9 \%$ of adult recipients [44] with an increased incidence in children and grafts where size discrepancy exists between donor artery and native vessel $[19,48]$. Operative exploration is the gold standard for diagnosis with surgical treatment including thrombectomy with anastomotic revision, aortic conduit graft, or arterial/vein interposition graft [49•]. Endovascular treatment using intra-arterial thrombolysis, embolization, or percutaneous transluminal angioplasty has lower success rates but may be appropriate in some patients [50, 51]. Early detection and management are essential for improved treatment outcomes and better prognosis [47•]. Lack of improvement after $48 \mathrm{~h}$ warrants consideration of retransplantation.

Portal vein thrombosis in adult recipients is rare but catastrophic, with an estimated incidence of $\sim 2 \%$ [52]. It is often predisposed to by anastomotic issues. Presentation may involve massive transaminitis with ascites, gastric, and variceal bleeding [1]. Treatment requires expedited surgical thrombectomy, though emergent retransplantation may be necessary. Interventional thrombolysis carries high risk of reocclusion and anastomotic injury [52].

\section{Rejection}

The advent of CNI's along with advances in immunosuppression has reduced the incidence of morbidity and mortality from graft rejection. Rejection usually presents following discharge from the ICU and is classified as acute, late acute, and chronic [53]. Acute rejection is most common with an incidence ranging from 25 to $46 \%$ within the first year [53, 54]. Risk factors include inadequate immunosuppression, underlying autoimmune disease, female recipients of male donors, and fewer human leukocyte antigen (HLA) matches at the DR locus [53].

Patients initially present with elevated liver enzymes followed later by nonspecific clinical signs such as fever, malaise, abdominal pain, jaundice, and decreased bile. Exclusion of hepatic artery or portal vein thrombosis, biliary leak, CMV infection and delayed graft function are necessary. Percutaneous liver biopsy is the gold standard for diagnosis with some centers instigating protocol biopsies allowing for early management. Treatment involves optimization of maintenance immunosuppression with an increased dose and additional agent. High-dose corticosteroids are often commenced. If no improvement is seen, an IL-2 receptor antagonist may be commenced [54]. In severe treatment-resistant disease, retransplantation may be necessary.

\section{Biliary Complications}

Biliary complications are the most common technical complication following transplant with increased incidence in living donor transplants. Presentations include lack of bile, elevated cholestatic enzymes, and leukocytosis [19]. Pathology often relates to anastomotic leak or obstruction from stenosis or stricture. Cholangiography is required for diagnostic confirmation and assessment of severity. Treatment depends on clinical status and may be conservative, surgical (repair) or interventional (ERCP or percutaneous drainage/stenting/dilation).

\section{Respiratory Dysfunction}

Respiratory dysfunction following OLT carries implications of prolonged ventilation, graft dysfunction, increased length of ICU stay, and patient morbidity and mortality. Preoperative predisposing factors include increased disease 
severity, high-grade encephalopathy, large volume ascites, pleural effusions, severe pulmonary artery hypertension, and pre-existing hypoxia from ventilation/perfusion mismatch or hepatopulmonary syndrome [55•]. Intraoperatively, a large volume transfusion, positive fluid balance, and excessive use of fresh frozen plasma or platelets may contribute to pulmonary edema and potential lung injury [56]. Postoperatively, respiratory dysfunction may arise from atelectasis, pleural effusion, volume overload, infection, adult respiratory distress syndrome (ARDS), and transfusion-related acute lung injury [55॰].

Management of respiratory dysfunction is generally supportive. Specific causes are targeted where possible. Patients with severe pulmonary artery hypertension may benefit from pulmonary vasodilators. Pulmonary edema warrants diuretic therapy and reduction in preload. Pneumonias require isolation of responsible microorganism with prompt instigation of appropriately targeted antibiotic therapy [55॰]. Ventilation parameters should be tailored to the patients underlying dysfunction. For pulmonary edema and ARDS, low tidal volumes $(6 \mathrm{~mL} / \mathrm{kg})$, avoidance of alveolar overdistension, and lower plateau airway pressures are beneficial [1]. PEEP should be set to improve oxygenation without restricting liver outflow. Debate exists as to whether high PEEP impairs liver blood outflow [1]. Saner et al., however, showed that PEEP up to $15 \mathrm{~cm} \mathrm{H}_{2} \mathrm{O}$ did not decrease hepatic vein outflow or portal blood flow [57].

Prolonged mechanical ventilatory support predisposes to ventilator-associated pneumonia, muscle deconditioning, and tracheal injury [30]. In the general ICU population, ventilator weaning strategies reduce cost and length of stay, improving 1-year survival [30]. These protocolized strategies should be applied to ventilated liver transplant recipients. Examples include spontaneous breathing periods and interruption of sedation [1]. Transition to noninvasive ventilation should be considered in patients who may not be immediately successful with extubation [55॰].

\section{Renal Dysfunction}

The incidence of renal dysfunction following liver transplant ranges widely due to differences in criteria and definition. Presentation may be acute or chronic with acute renal failure being a common complication following liver transplant. Much has been written on defining acute kidney injury (AKI) with the Risk, Injury, Failure, Loss, and EndStage Kidney Disease (RIFLE) criteria [58]. Etiologies for renal failure are complex and multi-factorial, contributed to by pre-existing renal dysfunction, hepatorenal syndrome, intraoperative course, graft dysfunction, infection, immunosuppressants, and prolonged use of vasoactive agents [59•]. Isolated liver transplant treats hepatorenal syndrome while combined kidney liver transplant may treat advanced irreversible disease [60].

A systematic approach assessing pre-renal, intrinsic, and post renal causes is useful when evaluating renal dysfunction. Serum creatinine-based estimates of renal function have proven inaccurate in the setting of end-stage liver disease, because creatinine elevations are often delayed [61]. The close monitoring of urine output, fluid balance, and hemodynamic parameters is critical in anticipating potential renal dysfunction in the ICU. Oliguria is an early warning of renal dysfunction; however, assessment is complex and must take into account multiple clinical and biochemical factors. Nephrotoxic drugs such as the CNIs must be used with appropriate dosing and therapeutic levels, with alternatives considered for high-risk patients.

Refractory volume overload and electrolyte disturbances (refractory hyperkalemia, hyper uremia, and metabolic acidosis) are the most common indications for RRT [30]. Continuous lactate-free RRT is most often undertaken because it causes less fluid shifts and hemodynamic instability. RRT is predictive of worse outcome, with longer ICU stay and higher mortality rates [62, 63].

\section{Neurological Dysfunction}

Neurological complications following liver transplantation occur at an incidence of $\sim 20 \%$ [64]. An increased frequency is observed in deceased donor versus living donor transplants [65]. Presentation is usually early after surgery and often in the ICU.

Encephalopathy is the most common CNS complication following liver transplantation [66]. With a spectrum from drowsiness and confusion, to delirium and coma, the etiology usually relates to excess ammonia. Improvement should be seen following transplantation. If mental status does not improve, investigation of graft function is warranted.

Seizures are the second most common neurological complication after liver transplant. Usually tonic-clonic, they relate to encephalopathy, immunosuppressant therapy, infection, metabolic derangements, and hypoxic-ischemic injury [67]. Correction of the underlying cause is necessary. Drug treatment and prophylaxis must consider interactions with immunosuppressants given their cytochrome P450 metabolism. Leviteracetam should be considered first line with phenytoin as an alternative [67].

Immunosuppression-related neurotoxicity may occur through use of tacrolimus. Presentations include tremor, headache, seizures, paresthesias, polyneuropathy, and myopathy [65]. Posterior-reversible encephalopathy syndrome from CNI insult to the blood brain barrier has also been described [68]. Treatment involves dose reduction, brief cessation, or interchange with CNI-sparing regimens. 
Transplant recipients are at risk of CNS infections due to immunosuppression. This usually occurs late after liver transplant. Incidence is rare, but mortality is high. Common organisms include Listeria monocytogenes, Aspergillus fumigatus, and Cryptococcus neoformans [69]. Viral infections occur due to herpes simplex virus and cytomegalovirus, but the incidence has declined with the use of antiviral prophylaxis.

\section{Special Circumstances}

\section{Living Donor Liver Transplant Recipients}

A number of differences exist in the ICU management of living donor liver recipients as compared to deceased donor recipients. Right lobe grafts are most commonly used and involve a more complex, technically challenging surgery. Greater caution is required in IV fluid replacement as size differentials increase susceptibility to graft congestion, and "small for size syndrome" may occur. The incidence of biliary and hepatic artery complications is higher, carrying greater risk of reoperation. Vigilance on the part of the intensivist is therefore necessary for early detection and appropriate management.

\section{Transplantation for Acute Liver Failure}

Liver transplant recipients with acute liver failure experience longer ICU lengths of stay with increased morbidity and mortality [70]. Postoperative ventilation is standard as patients may have high-grade encephalopathy and cerebral edema necessitating intracranial pressure monitoring, often with the added challenges of coagulopathy, portopulmonary hypertension, and hemodynamic instability. Patients also have greater risk of complications with a higher requirement for RRT and increased risk of infection and rejection [71].

\section{Conclusion}

The ICU course following liver transplantation remains a critical step following surgery. Despite the challenges of increasing disease severity, an older comorbid population and increased use of marginal donors, ICU lengths of stay, readmissions, and overall morbidity and mortality have greatly improved. The majority of admissions are indeed uneventful. While advances in surgical technique, perioperative management, and immunosuppression regimens are to be credited, so too should the role of the intensivist in adopting a thorough patient assessment, with anticipation and early management of complications. Paramount to this approach is involvement of a multidisciplinary team, utilizing a patient-focused approach and institution-specific protocols guiding management.

Acknowledgments The Authors wish to thank Dr. Stephen Streat for his help with review of the article.

\section{Compliance with Ethics Guidelines}

Conflict of Interest Thomas M.A. Fernandez and Paul J. Gardiner declare that they have no conflict of interest.

Human and Animal Rights and Informed Consent This article does not contain any studies with human or animal subjects performed by any of the authors.

\section{References}

Papers of particular interest, published recently, have been highlighted as:

- Of importance

1. McKenna GJ, Klintmalm GBG. Postoperative intensive care management in adults. In: Busuttil RW, Klintmam GBG, editors. Transplantation of the liver, vol. 69. Philadelphia: Saunders; 2015. p. 865-94.

2. Cotler SJ. Liver transplantation: donor selection. http://www. uptodate.com/contents/liver-transplantation-donor-selection. Accessed 28 July 2015.

3. Feng S, Goodrich NP, Bragg-Gresham AL, et al. Characteristics associated with liver graft failure: the concept of a donor risk index. Am J Transplant. 2006;4:783-9.

4. Mandell MS, Stoner TJ, Barnett R, et al. A multicentre evaluation of safety of early extubation in liver transplant recipients. Liver Transpl. 2007;13:1557-63.

5. Mandell MS, Campsen J, Zimmerman M, et al. The clinical value of early extubation. Curr Opin Organ Transplant. 2009;14: 297-302.

6. Glanemann M, Hoffmeister R, Neumann U, et al. Fast tracking in liver transplantation which patient benefits from this approach? Transplant Proc. 2007;39:535-6.

7. Biancofiore G, Romanelli AM, Bindi ML, et al. Fast track in liver transplantation: 5 year's experience. Eur J Anesthesiol. 2005;22: 584-90.

8. Mandell MS, Lezotte D, Kam I, et al. Reduced use of intensive care after liver transplantation: influence of early extubation. Liver Transpl. 2002;8:676-81.

9. Mandell MS, Hang Y. Pro: early extubation after liver transplantation. Cardiothorac Vasc Anesth. 2007;21(5):752-5.

10. Steadman R. Con: immediate extubation for liver transplantation. Cardiothorac Vasc Anesth. 2007;21(5):756-7.

11. Liu LL, Niemann CU. Intraoperative management of liver transplant patients. Transplant Rev. 2011;25:124-9.

12. Costa MG, Chiarandini P, Della Roca G. Hemodynamics during liver transplantation. Transplant Proc. 2007;39:1871-3.

13. Kumar A, Anel R, Bunnell E, et al. Pulmonary artery occlusion pressure and central venous pressure fail to predict ventricular filling volume, cardiac performance, or the response to volume infusion in normal subjects. Crit Care Med. 2004;32:691-7.

14. Bossert T, Gummert JF, Bittner HB, et al. Swan-Ganz catheterinduced severe complications in cardiac surgery: right ventricular 
perforation, knotting and rupture of a pulmonary artery. J Card Surg. 2006;21(3):292-5.

15. Michard F, Teboul JL. Predicting fluid responsiveness in ICU patients: a critical analysis of the evidence. Chest. 2002;121(6): 2000-8.

16. - Marik PE. Obituary: pulmonary artery catheter 1970 to 2013. Ann Intensive Care. 2013;3:38-43. Outline of the history of the $P A C$ with review of limitations leading to decline in its use.

17. Buais M, Nouette-Gaulain K, Cottenceau V, et al. Cardiac output measurement in patients undergoing liver transplantation: pulmonary artery catheter versus uncalibrated arterial pressure waveform analysis. Anesth Analg. 2008;106:1480-6.

18. Munshi L, Scales DC, Granton JT. The transplant patient. In: Hall JB, Schmidt GA, Kress JP, editors. Principles of critical care, vol. 115. New York: McGraw Hill; 2015. p. 1097.

19. Feltracco $\mathrm{P}$, Barbieri S, Galligioni $\mathrm{H}$, et al. Intensive care management of liver transplanted patients. World $\mathrm{J}$ Hepatol. 2011;3(3):61-71

20. Mandell MS, Tsou MJ. The development of perioperative practices for liver transplantation: advances and current trends. J Chin Med Assoc. 2008;71:435-41.

21. Levy MF, Greene L, Ramsay MA, et al. Readmission to the intensive care unit after liver transplantation. Crit Care Med. 2001;29:18-24.

22. Ruiz R, Trotter J, Klintmalm GBG. Postoperative management beyond the intensive care unit: adults. In: Busuttil RW, Klintmam GBG, editors. Transplantation of the liver. Philadelphia: Saunders; 2015. p. 905-15.

23. O'Grady JG, Hardy P, Burroughs AK, et al. Randomized controlled trial of tacrolimus versus microemulsified cyclosporin in liver transplantation: the TMC randomized controlled trial. Lancet. 2002;360:1119.

24. Haddad EM, McAlister VC, Renouf E, et al. Cyclosporin vs tacrolimus for liver transplanted patients. Cochrane Database Syst Rev. 2006;4:CD005161.

25. McKenna GJ, Klintmalm GBG. Induction and maintenance of immunosuppression. In: Busuttil RW, Klintmalm GBG, editors. Transplantation of the liver. Philadelphia: Saunders; 2015. p. $1264-88$.

26. Lake JR. Immunosuppression and outcomes of patients transplanted for hepatitis C. J Hepatol. 2006;44:627.

27. Klintmalm GB, Davis GL, Teperman L, et al. A randomized, multicentre study comparing steroid-free immunosuppression and standard immunosuppression for liver transplant recipients with chronic hepatitis C. Liver Transpl. 2011;17:1394.

28. http://www.accessdata.fda.gov/drugsatfda_docs/label/2008/021 083s033,021110s043lbl.pdf. Accessed 1 Aug 2015.

29. Sussman NL, Vierling JM. Liver transplantation in adults: overview of immunosuppression. http://www.uptodate.com/contents/ liver-transplantation-in-adults-overview-of-immunosuppression. Accessed 28 July 2015.

30. Razonable RR, Finday JY, O'Riordan A, et al. Critical care issues in patients after liver transplantation. Liver Transpl. 2011;17:511-27.

31. Clark NM, Cotle SJ. Infectious complications in liver transplantation. http://www.uptodate.com/contents/infectious-complicationsin-liver-transplantation. Accessed 28 July 2015.

32. Niemann CU, Kramer DJ. Transplant critical care: standards for intensive care of the patient with liver failure before and after transplantation. Liver Transpl. 2011;17:485-7.

33. Figueriredo F, Dickson ER, Pasha T, et al. Impact of nutritional status on outcomes after liver transplantation. Transplantation. 2000;70:1347-52.

34. Sanchez AJ, Aranda-Michel J. Nutrition for the liver transplant patient. Liver Transpl. 2006;12:1310-6.
35. Hasse J. Liver transplantation: the benefits of nutrition therapy in the liver transplant patient. In: Klintmalm G, editor. Recent developments in transplantation medicine, vol. III. Glenview: Physicians and Scientists Publishing Company; 1996. p. 81-100.

36. Busani S, Semeraro G, Cantaroni C, et al. Recombinant activated factor VII in critical bleeding after orthotopic liver transplantation. Transplant Proc. 2008;40:1989-90.

37. - Meybohm P, Zacharowski K, Weber CF. Point-of-care coagulation management in intensive care management. Critical Care. 2013;17:218. A Review of point of care tests with a management approach to the patient with coagulopathy. Specific description of patient groups relevant to the ICU.

38. Wang SC, Shieh JF, Chang KY, Chu YC, Liu CS, Loong CC. Thromboelastography-guided transfusion decreases intraoperative blood transfusion during orthotopic liver transplantation: randomized clinical trial. Transplant Proc. 2010;42:2590-3.

39. Gorlinger K, Fries D, Dirkmann D, et al. Reduction of fresh frozen plasma requirements by perioperative point-of-care coagulation management with early calculated goal-directed therapy. Transfus Med Hemother. 2012;39:104-13.

40. Krzanicki D, Sugavanam A, Mallet S. Intraoperative hypercoagulability during liver transplantation as evidenced by thromboelastography. Liver Transpl. 2013;19:852-61.

41. Schumann R. Intraoperative resource utilization in anesthesia for liver transplantation in the United States: a survey. Anesth Analg. 2003;97:21-8.

42. - Saner FH, Gieseler RK, Akiz H, et al. Delicate balance of bleeding and thrombosis in end stage liver disease and liver transplantation. Digestion. 2013;88:135-144. A review of the complexities of coagulation in the cirrhotic patient. Outlines developments and treatments for hemostatic disorder in cirrhotics.

43. Onaca N, Klintmalm GBG. Clinical management of necrotic liver before and after transplantation. In: Busuttil RW, Klintmam GBG, editors. Transplantation of the liver. Philadelphia: Saunders; 2015. p. 811-7.

44. Petrowsky H, Busuttil RW. Graft failure. In: Busuttil RW, Klintmam GBG, editors. Transplantation of the liver. Philadelphia: Saunders; 2015. p. 960-74.

45. Uemura T, Randell HB, Sanchez EQ, et al. Liver transplantation for primary nonfunction: analysis of a 20 year single-centre experience. Liver Transpl. 2007;13(2):227-33.

46. Chen $\mathrm{XB}, \mathrm{Xu} \mathrm{MQ}$. Primary graft dysfunction after liver transplantation. Hepatobiliary Pancreat Dis Int. 2014;13(2):125-37.

47. - Mourad MM, Liossis C, Gunson BK, et al. Etiology and management of hepatic artery thrombosis after adult liver transplantation. Liver Transpl. 2014;20(6):713-23. A retrospective review of 102 episodes of hepatic artery thrombosis assessing risk factors, etiology, management and survival.

48. Crippin J. Pathogenesis of organ dysfunction. In: Norman DJ, Suki WN, editors. Primer on transplantation. Mt Laurel: American Society of Transplant Physicians; 1998. p. 321-7.

49. - Chen J, Weinstein J, Black S, et al. Surgical and endovascular treatment of hepatic arterial complications following transplant surgery. Clinical Transpl. 2014;28(12):1305-12. A review of the surgical and interventional radiological options for treatment of hepatic artery thrombosis.

50. Singhal A, Stokes K, Sebastian A, et al. Endovascular treatment of hepatic artery thrombosis following liver transplantation. Transpl Int. 2010;23(3):245-56.

51. Abdelaziz O, Hosny K, Amin A, et al. Endovascular management of early hepatic artery thrombosis after living donor liver transplantation. Transpl Int. 2012;25(8):847-56.

52. Duffy JP, Hong JC, Farmer EG, et al. Vascular complications of orthotopic liver transplantation: experience in more than 4200 patients. J Am Coll Surg. 2009;208(5):896-903. 
53. Shaik I, Carmody IC, Chen PW. Treatment of acute and chronic rejection. In: Busuttil RW, Klintmalm GBG, editors. Transplantation of the liver, vol. 93. New York: Elsevier; 2015. p. 1317-28.

54. Parekh J, Ascher NL, Roberts J. Rejection after transplantation. In: Busuttil RW, Klintmalm GBG, editors. Transplantation of the liver, vol. 87. New York: Elsevier; 2015. p. 1212-26.

55. - Feltracco P, Carollo C, Barbieri S, et al. Early respiratory complications after liver transplantation. World J Gastroenterol. 2013;19(48):9271-81. A Comprehensive review of the risk factors, causes and management strategies for respiratory complications specific to the liver transplant recipient in the ICU.

56. De Gasperi A, Feltracco P, Ceravola E, et al. Pulmonary complications in patients receiving a solid-organ transplant. Curr Opin Crit Care. 2014;20:411-9.

57. Saner FH, Olde Damink SW, Pavlakovic G, et al. How far can we go with positive end-expiratory pressure (PEEP) in liver transplant patients? J Clin Anesth. 2010;22:104-9.

58. Bellomo R, Ronco C, Kellum JA, et al. Acute renal failuredefinition, outcome measures, animal models, fluid therapy and information technology needs: the second international consensus conference of the acute dialysis quality initiative (ADQI) group. Crit Care. 2004;8(4):R204-12.

59. - Weber ML, Ibrahim HN, Lake JR. Renal dysfunction in liver transplant recipients: evaluation of the critical issues. Liver Transpl. 2012;18:1290-301. Comprehensive review of the assessment and causes of renal dysfunction in the liver transplant population.

60. Pham PT, Parke C, Allamezadeh R, et al. Renal failure in adults. In: Busuttil RW, Klintmalm GBG, editors. Transplantation of the liver, vol. 74. New York: Elsevier; 2015. p. 934-59.

61. Francoz C, Prie D, Abdelrazek W, et al. Inaccuracies of creatinine and creatinine-based equations in candidates for liver transplantation with low creatinine: impact of the model for endstage liver disease score. Liver Transpl. 2010;16(10):1169-77.

62. Fraley DS, Burr R, Bernardini J, et al. Impact of acute renal failure on mortality in end-stage liver disease with or without liver transplantation. Kidney Int. 1998;4:518-24.

63. Faenza S, Santoro A, Mancini E, et al. Acute renal failure requiring renal replacement therapy after orthotopic liver transplantation. Transpl Proc. 2006;38:1141-2.

64. Pustavoitau A, Bhardwaj A, Stevens R, et al. Neurological complications of transplantation. $\mathrm{J}$ Intensive Care Med. 2011;26:209-22.

65. Saner FH, Gu Y, Minouchehr S. Neurological complications after cadaveric and living donor liver transplantation. J Neurol. 2006;253:612-7.

66. Marco S, Cecilia F, Patrizia B. Neurologic complications after solid organ transplantation. Transpl Int. 2009;22:269-78.

67. Guarino M, Benito-León J, Decruyenaere J, et al. Neurological problems in liver transplantation. In: Gilhus NE, Barnes MP, Brainin M, editors. European handbook of neurological management, vol. 1. 2nd ed. Oxford: Wiley-Blackwell; 2011. p. 491-9.

68. Cruz RJ Jr, DiMartini A, Akhavanheidari M, et al. Posterior reversible encephalopathy syndrome in liver transplant patients: clinical presentation, risk factors and initial management. Am J Transplant. 2012;8:2228-36.

69. Guarino M, Benito-Leon J, Decruyenaere J, et al. EFNS guidelines on management of neurological problems in liver transplantation. Eur J Neurol. 2006;13:2-9.

70. O'Grady JG. Postoperative issues and outcome for acute liver failure. Liver Transpl. 2008;14:S97-101.

71. O'Grady JG. Transplantation for fulminant hepatic failure. In: Busuttil RW, Klintmam GBG, editors. Transplantation of the liver, vol. 69. Philadelphia: Saunders; 2015. p. 865-94. 